ARTICLE

Received 7 Oct 2014 | Accepted 4 Jun 2015 | Published 15 Jul 2015

\title{
SUMOylation of synapsin la maintains synaptic vesicle availability and is reduced in an autism mutation
}

Leo T.-H. Tang ${ }^{1}$, Tim J. Craig ${ }^{1, \star} \&$ Jeremy M. Henley ${ }^{1, \star}$

Synapsins are key components of the presynaptic neurotransmitter release machinery. Their main role is to cluster synaptic vesicles (SVs) to each other and anchor them to the actin cytoskeleton to establish the reserve vesicle pool, and then release them in response to appropriate membrane depolarization. Here we demonstrate that SUMOylation of synapsin la (Synla) at K687 is necessary for Synla function. Replacement of endogenous Synla with a non-SUMOylatable mutant decreases the size of the releasable vesicle pool and impairs stimulated SV exocytosis. SUMOylation enhances Synla association with SVs to promote the efficient reclustering of Synla following neuronal stimulation and maintain its presynaptic localization. The A548T mutation in Synla is strongly associated with autism and epilepsy and we show that it leads to defective Synla SUMOylation. These results identify SUMOylation as a fundamental regulator of Synla function and reveal a novel link between reduced SUMOylation of Synla and neurological disorders.

\footnotetext{
${ }^{1}$ School of Biochemistry, Medical Sciences Building, University of Bristol, University Walk, Bristol BS8 1TD, UK. * These authors contributed equally to this work. Correspondence and requests for materials should be addressed to T.J.C. (email: Tim.Craig@bristol.ac.uk) or to J.M.H. (email: j.m.henley@bristol.ac.uk).
} 
T he coordinated release of neurotransmitter from synaptic vesicles (SVs) is fundamental to synaptic function and plasticity. It is generally accepted that SVs are organized into distinct pools at the presynapse including the readily releasable pool that is synchronously released immediately following stimulation and the reserve pool (RP), that is mobilized during prolonged stimulations. Vesicles that are refractory to release are often referred to as the resting pool ${ }^{1}$. SynIa maintains the RP of SVs by tethering them to each other and to the presynaptic actin cytoskeleton near to presynaptic release sites. Presynaptic depolarization causes SynIa phosphorylation through the CamKII and PKA pathways, which decreases binding affinity and releases the tethered SVs allowing them to move to the active $z^{2} e^{2-4}$. On the other hand, phosphorylation by other kinases such as c-Src and cdk5 alters the distribution of SVs between different pools ${ }^{5,6}$.

SUMOylation is the covalent attachment of the small ubiquitin-like modifier (SUMO) to lysine residues on target proteins. In neurons, SUMOylation a key regulator of transcription and extranuclear SUMOylation plays fundamentally important roles in synaptic function ${ }^{7-10}$ and neuroprotective responses to severe stressors ${ }^{11}$. Recently, we reported that SUMOylation of RIM1 $\alpha$ is required for normal SV exocytosis, demonstrating that SUMOylation also has a role in presynaptic regulation ${ }^{12}$.

Here we show that SUMOylation of SynIa at K687 enhances binding to SVs and facilitates synapsin-mediated SV clustering and anchoring. Replacement of endogenous SynIa with a nonSUMOylatable K687R mutant in neurons reduces the number of releasable SVs and impairs exocytosis. Furthermore, we demonstrate that an A548T mutation in SynIa, which has been linked to autism spectrum disorder (ASD) and epilepsy, reduces SynIa SUMOylation and mirrors the functional defect of nonSUMOyatable SynIa, suggesting a possible causal link between dysfunctional SynIa SUMOylation and neurological disorders.

\section{Results and Discussion}

Synapsin Ia is a SUMO substrate. To validate SynIa as a SUMO-1 substrate, we used a SUMOylation assay system in which we exogenously expressed HA-SynIa, Flag-Ubc9 and either YFP-SUMO or non-conjugatable YFP-SUMO- $\Delta$ GG in N2A cells. A SUMOylated SynIa band was detected in the cells expressing YFP-SUMO but not YFP-SUMO- $\Delta$ GG (Fig. 1a, upper panel). This band was also present in green fluorescent protein (GFP)trap pull downs of YFP-SUMO from the N2A lysate, further confirming it corresponds to SUMOylated SynIa (Fig. 1a, lower panel). We attribute the presence of an equal density unmodified SynIa band to dimerization of SynIa and SynIa-SUMO.

Ubc9 binding to the target protein is a critical step in SUMOylation. Consistent with SynIa being a robust SUMO substrate, endogenous SynIa in cultured cortical neuronal lysates is strongly retained by GST-Ubc9 pull downs (Fig. 1b).

Importantly, we also detected endogenous SUMOylated SynIa in neurons by anti-SUMO co-immunoprecipitation from whole-brain lysate. As expected, anti-SynI antibody immunoblots of the immunoprecipitated protein revealed a $\sim 110-\mathrm{kDa}$ band corresponding to SUMOylated SynIa. Critically, this band was removed by treatment with the deSUMOylating enzyme SENP1 (Fig. 1c). Again, we attribute the presence of unmodified SynIa in this co-immunoprecipitation to dimerization.

SynIa is a multidomain protein; therefore, to define the SUMOylation site(s), we first generated and expressed SynIa mutants lacking the $\mathrm{E}$ domain $(\Delta \mathrm{E})$ or both $\mathrm{D}$ and $\mathrm{E}$ domains $(\triangle D E)$ in HEK293T cells and performed GST-Ubc9 pull-down assays (Fig. 1d). Removal of the E domain of SynIa greatly decreased the interaction with GST-Ubc9, and additional deletion of the D domain completely abolished the interaction (Fig. 1d). These data indicate that Ubc9 binds mainly to a region within the E domain, but may also have some interaction within the D domain.

Consistent with this, the $\mathrm{E}$ domain contains the SUMO consensus sequence VKAE at residues 686-689. Mutation of the Val686 or Glu689 residues flanking the target lysine to Ala disrupted the SynIa-GST-Ubc9 interaction (Fig. 1e) and mutating Lys687 to Arg completely prevented SynIa SUMOylation (Fig. 1f).

The $\mathrm{E}$ domain is involved in dimerization, vesicle clustering, presynaptic targeting, cytoskeleton interaction and vesicle release $^{13,14}$. All synapsin a-isoforms (SynIa, SynIIa and SynIIIa) contain highly conserved $\mathrm{E}$ domains $\mathrm{s}^{15,16}$ and SynIIa could also be SUMOylated in N2A cells, even though the consensus sequence is AKAE in this protein (Supplementary Fig. 1a). As shown in Fig. 1e, V686A inhibited Ubc9 binding to SynIa in HEK293T cells. However, in N2A cells, this mutation could still be SUMOylated (Supplementary Fig. 1b), indicating that an additional factor present in this neuronal cell line, presumably an E3 ligase, is required to enhance SUMOylation. Sequence alignment shows that K687 is also conserved across a-isoforms of synapsins in vertebrates (Supplementary Fig. 1c). However, despite its importance, the molecular mechanisms underlying the function of the E domain is not well characterized.

Preventing SynIa SUMOylation causes defective SV exocytosis. The SUMO consensus sequence VKAE is present in SynIa across most vertebrate species consistent with SUMOylation playing an important role in SynIa function throughout evolution. We therefore tested the effect of acutely reducing endogenous SynIa by short hairpin RNA (shRNA) knockdown and replacement with shRNA-insensitive wild-type SynIa or the nonSUMOylatable mutant. Transfection of hippocampal neurons with SynI shRNA decreased in SynI levels to $50 \%$ of control levels. Transfection with shRNA-resistant wild-type or K687R SynIa constructs restored SynI to near-endogenous levels (Supplementary Fig. 2).

We investigated the dynamics of SV exocytosis using the genetically encoded reporter Synaptophysin-pHluorin (SypHy) ${ }^{17}$. Hippocampal neurons transfected with both SypHy and knockdown-rescue constructs were subjected to 600 action potentials at $20 \mathrm{~Hz}$ using electrical field stimulation to evoke SV exocytosis of all releasable vesicles (that is, readily releasable pool and RP). These experiments were performed in the presence of the proton pump inhibitor bafilomycin1A to prevent reacidification of endocytosed SVs so that each SV is counted only once on first-time release ${ }^{18}$. The SypHy fluorescence was expressed as the change in fluorescence normalized against the basal signal $\left(\Delta F / F_{0}\right)$, presented in Fig. 2a,b. To assess the average size of the SV pool in the presynapse, the mean level of $\Delta F / F_{0}$ at the end of the experiment (during $50 \mathrm{mM} \mathrm{NH}_{4} \mathrm{Cl}, F_{\text {max }}$ ) was calculated and is presented in Fig. 2c. No significant difference was observed between any conditions, indicating that the knockdown of Syn1 or replacement with a non-SUMOylatable mutant did not change the total SV pool. To selectively assess the size and dynamics of the releasable pool, we normalized the change in fluorescence against $F_{\max }\left(\Delta F / F_{\max }\right)$. Consistent with previous reports using knockout mice, acute shRNA-mediated knockdown of SynI significantly decreased the total level of SV exocytosis, measured by the average $\Delta F / F_{\max }$ after stimulation, indicating a reduction in releasable vesicle pool size ${ }^{19-21}$. Importantly, a scrambled shRNA control had no effect (Supplementary Fig. 3a,b). Replacement of the knockdown with 
a

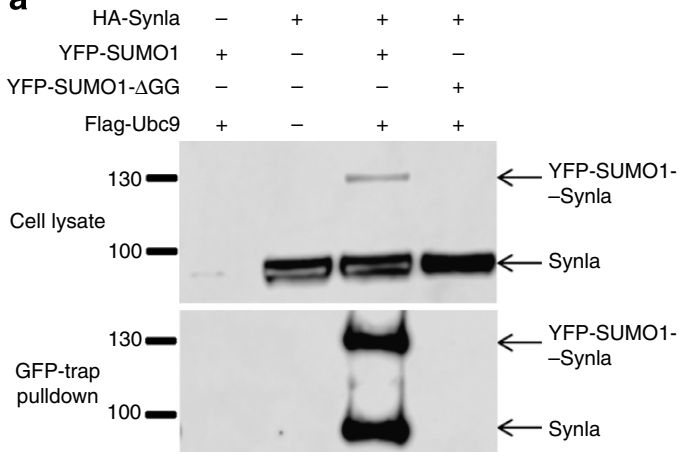

C

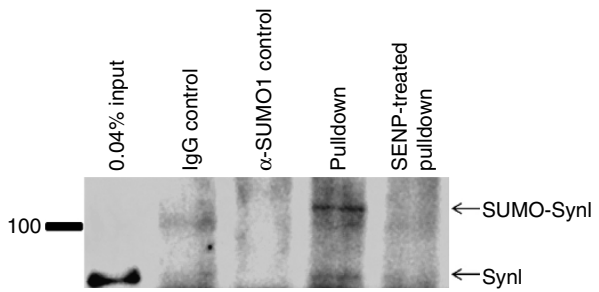

e

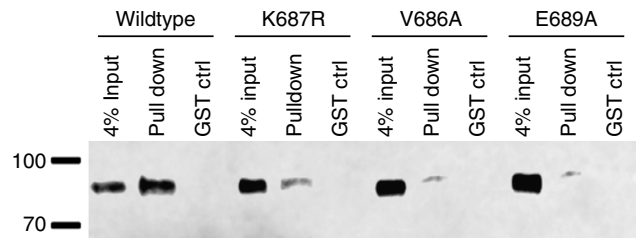

b

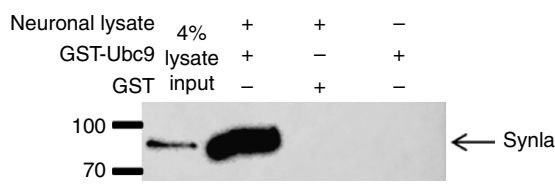

d

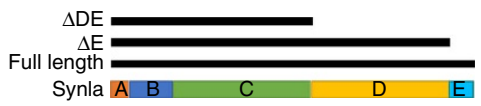

$\begin{array}{lllll}\text { Synla A } & B & C & D & E\end{array}$

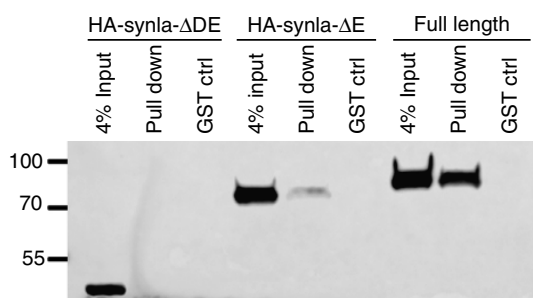

f

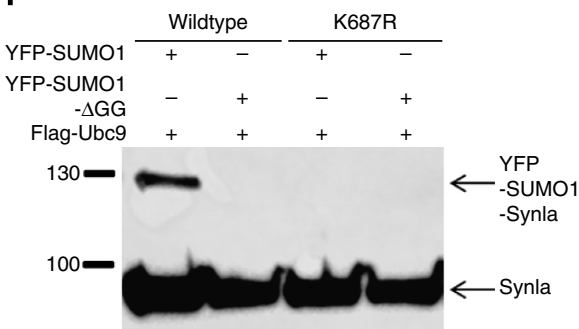

Figure 1 | Synla is SUMOylated at K687R. All experiments were performed at least three times with consistent results. (a) Representative anti-HA immunoblot showing that Synla is robustly SUMOylated. HA-Synla, Flag-Ubc9 and YFP-SUMO-1 were expressed in N2A cells and the lysate then subjected to GFP-trap pull down to purify YFP-SUMOylated proteins. (b) GST-pull-down assays showing that endogenous Synl from rat cortical neurons binds Ubc9. (c) Immunoprecipitation of endogenous SUMOylated Synla from rat brain. Whole rat brain was lysed under denaturing conditions and subjected to IP with anti-SUMO-1 antibody, then immunoblotted with anti-Synl antibody. IgG Control: mouse IgG was used instead of anti-SUMO-1; $\alpha$-SUMO-1 control: RIPA buffer was used instead of brain lysate. SENP-treated pull down: before the pull down, the lysate was supplemented with $25 \mathrm{nM}$ SENP (catalytic domain fragment) and incubated for $1 \mathrm{~h}$ at room temperature. (d) Full-length HA-Synla and truncation constructs lacking the $\mathrm{E}$ domain ( $\Delta \mathrm{E}$ ) or both the $\mathrm{D}$ and $\mathrm{E}$ domains $(\triangle \mathrm{DE}$ ) were expressed in HEK293T cells and the lysates subjected to GST-Ubc9 pull down. (e) Point mutations of residues in the SUMO consensus site in Synla prevent pull down by GST-Ubc9 in HEK293T cells. (f) K687 is the only SUMOylation site in Synla. HA-Synla WT or HA-Synla K687R were expressed with Flag-Ubc9 and YFP-SUMO-1 in N2A cells and lysates were immunoblotted with anti-HA antibody.

wild-type SynIa returned SV exocytosis to control levels. However, replacement with the SynI-K687R mutant did not rescue the normal release profile with levels of exocytosis indistinguishable from the knockdown neurons (Fig. 2d,e). This result cannot be attributed to a dominant-negative effect of K867R Syn1a, as expression of the K687R mutant in nonknockdown neurons did not cause any effect on SV exocytosis (Supplementary Fig. 3c,d). These data demonstrate that SynIa SUMOylation is required for normal levels of SV exocytosis.

This decrease in SV exocytosis could be mediated by reduced vesicle fusion or by a decrease in the number of releasable vesicles. We therefore assessed the rate of exocytosis by measuring the time constant of a single exponential growth

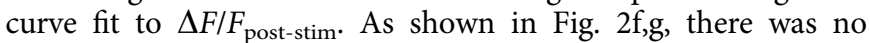
difference in the rates of vesicle fusion in neurons in which SynIa was knocked down, or knocked down and replaced with either wild-type SynIa or with the K687R mutant. Therefore, we attribute the decreased exocytosis in neurons expressing the non-SUMOylatable SynIa-K687R to a reduction in the size of the releasable vesicle pool, that is, fewer SVs available for release, rather than a defect in vesicle exocytosis.
We also tested if SV exocytosis is affected by a global decrease in SUMOylation by overexpressing the catalytic domain of SENP. Consistent with our results using non-SUMOylatable SynIa, overexpression of constitutively active SENP induced a decrease in the overall amount of SV exocytosis, whereas overexpression of the protease inactive mutant SENP C603S had no effect (Supplementary Fig. 4). This decrease was considerably more pronounced than molecular replacement with SynIa-K687R, suggesting that other proteins involved in SV regulation are also SUMO targets. While we cannot definitively exclude the possibility that downstream effects induced by decreased SUMOylation of non-synaptic proteins, for example, transcription factors, could contribute to this effect, our data are consistent with previous studies that identify other presynaptic SUMO substrates $^{12,22,23}$.

SUMOylation of SynIa enhances binding to SVs. It is well known that changes in the binding affinity of SynIa to SVs plays a major role in SV dynamics ${ }^{3}$. Therefore, we investigated whether SUMOylation of Syn1a affects its binding to SVs, using an 
a
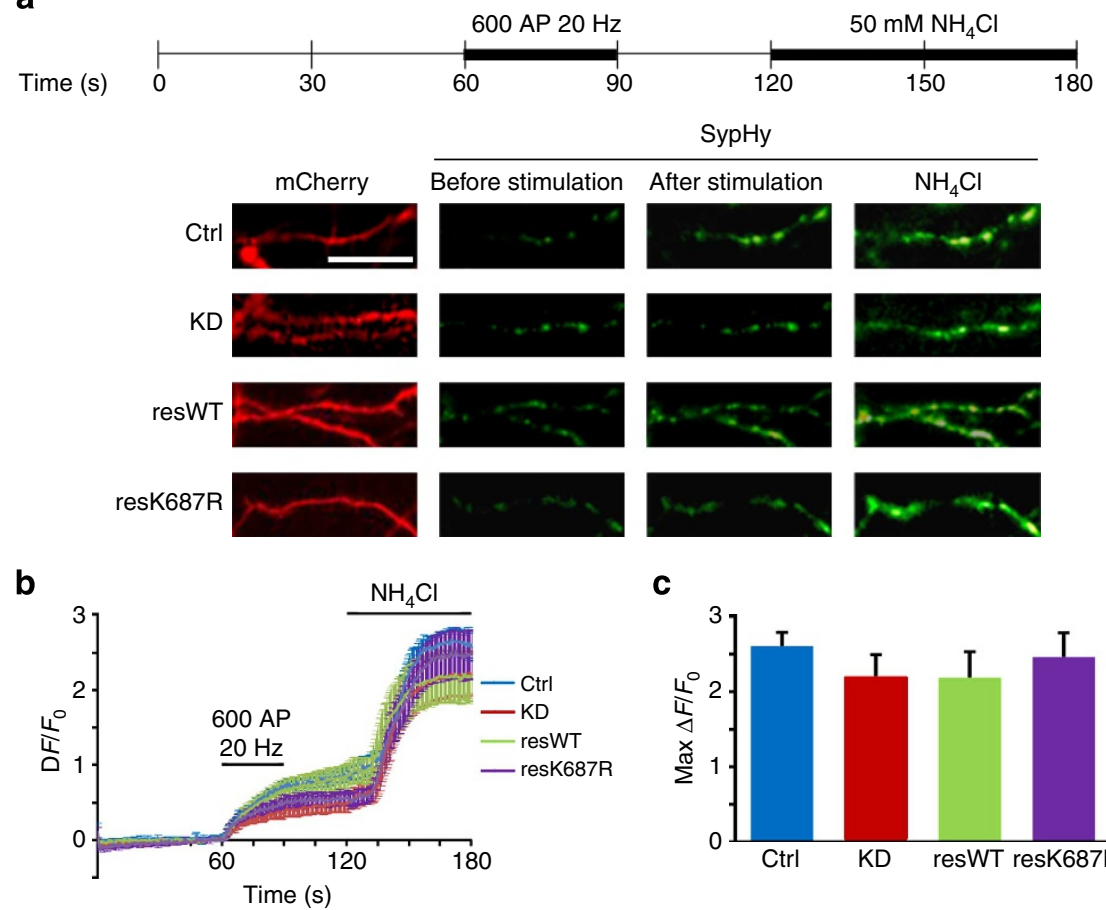

C

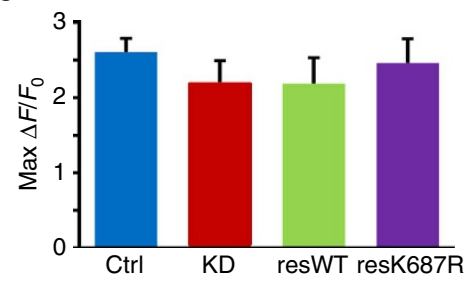

d

e
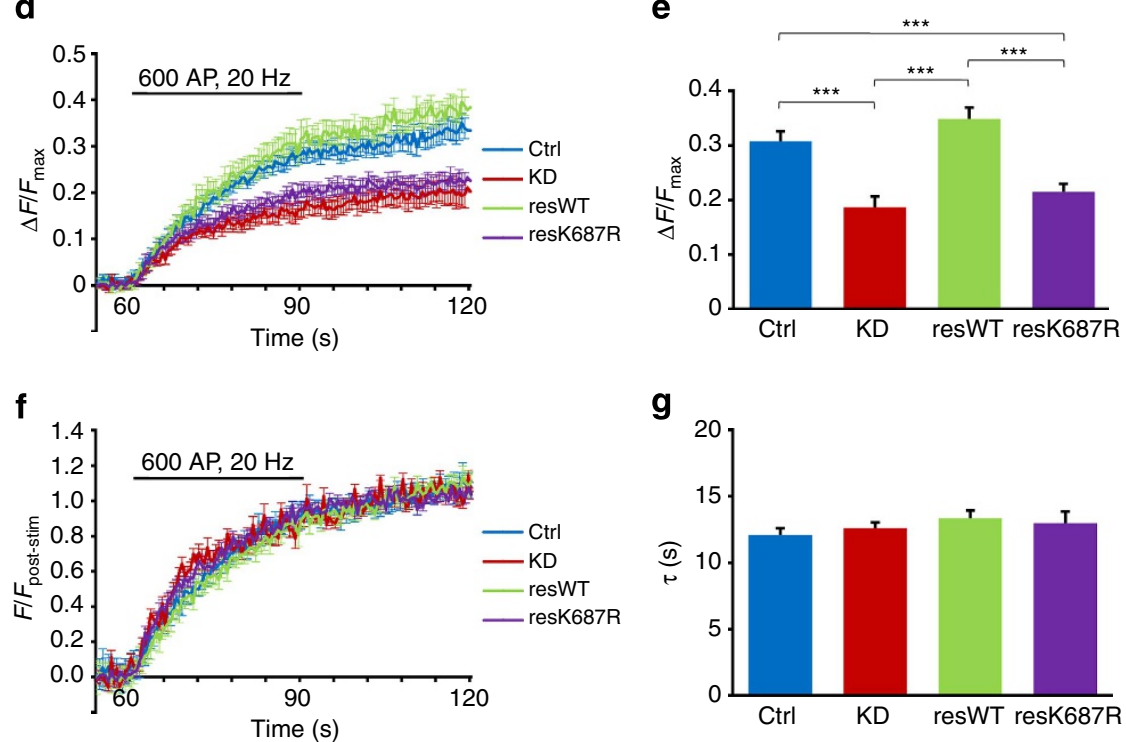

Figure 2 | SUMOylation of Synla regulates SV exocytosis. (a) SypHy assay on Synla knockdown and molecular replacement in DIV 15 hippocampal neurons, transfected and imaged live 5 days later. The bicistronic expression constructs incorporate mCherry to indicate transfection. The schematic shows the experimental time course and the images are representative of the SypHy signal before and after stimulation ( $t=0$ and $90 \mathrm{~s}$, respectively), and after $\mathrm{NH}_{4} \mathrm{Cl}$ perfusion $(t=180 \mathrm{~s}$ ). Control ( $\mathrm{Ctrl}, n=13)$, Knockdown (KD, $\left.n=12\right), \mathrm{KD}$-rescue WT (resWT, $\left.n=12\right)$ and KD-rescue K687R (resK687R, $n=12$ ). Scale bar, $10 \mu \mathrm{m}$. (b) Average traces of $\Delta F / F_{0}$ over the whole course of assay, plotted as mean \pm s.e.m. (c) Quantification of data shown in $\mathbf{b}$ plotted as the mean \pm s.e.m. of the average $\Delta F_{\max }(t=175-180 \mathrm{~s}) / F_{0}$, providing a measure of the total SV pool in the presynapse. One-way analysis of variance (ANOVA) showed no significant difference between any values. $n$ numbers as in a. (d) Replacement of endogenous Synla with Synla K687R causes defective exocytosis. SypHy fluorescence data were normalized to the maximum SypHy signal obtained after $\mathrm{NH}_{4} \mathrm{Cl}$ application $\left(\Delta F / F_{\text {max }}\right)$ and plotted as the mean \pm s.e.m. $n$ numbers as in a. (e) Quantification of data shown in d plotted as the mean \pm s.e.m. of the average $\Delta F / F_{\text {max }} t=90-100$ s, providing a measure of relative overall SV exocytosis against total SV pool size. ${ }^{\star \star \star} P<0.001$, one-way ANOVA, post hoc test with Bonferroni correction.

(f) Replacement of endogenous Synla with Synla K687R has no effect on the kinetics of SV release. Change in SypHy signal normalized against average signal after stimulation $\left(\Delta F / F_{\text {post-stim }}\right)$, plotted as mean \pm s.e.m. against time. $n$ numbers as in $\mathbf{a}$. $(\mathbf{g}) \Delta F / F_{\text {post-stim }}$ between 60 and $120 \mathrm{~s}$ were fitted to exponential curves and the time constant $\tau$ plotted as mean \pm s.e.m. Significance tested with one-way ANOVA, post hoc test with Bonferroni correction.

HA-His-tagged SynIa-DE domain fragment. SynIa-DE was co-expressed in bacteria with SUMOylation machinery with a Flag-tagged SUMOylation construct ${ }^{24,25}$. SUMOylated SynIa-DE was purified using metal-affinity purification followed of the SynIa followed by Flag-affinity purification (Fig. 3a). The purified, SUMOylated SynIa-DE was divided into equal aliquots, one of which was deSUMOylated with SENP1. The aliquots of SUMOylated and deSUMOylated SynIa-DE were then incubated 
with an enriched SV suspension from rat brain homogenate. The SVs were pelleted by ultracentrifugation and subjected to quantitative western blot analysis to determine the amount of bound SynIa-DE in SV fraction. SUMOylated SynIa-DE displayed significantly higher affinity binding to SVs than non-SUMOylated or deSUMOylated SynIa-DE (Fig. 3b,c). These results demonstrate that, opposite to CamKII phosphorylation of SynIa, SUMOylation increases the affinity of SynIa binding to SVs. Thus, these data support the hypothesis that SUMOylation of SynIa promotes SV binding and plays key roles in establishing and maintaining the reserve vesicle pool.

The generally accepted model of synapsin structure and function is that the $\mathrm{D}$ and $\mathrm{E}$ domains interact with SVs by binding to specific vesicle proteins, whereas the $\mathrm{A}$ and $\mathrm{C}$ domains

a

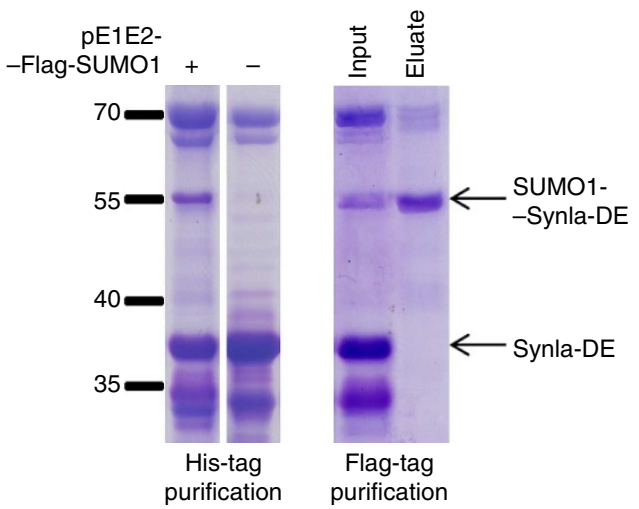

b

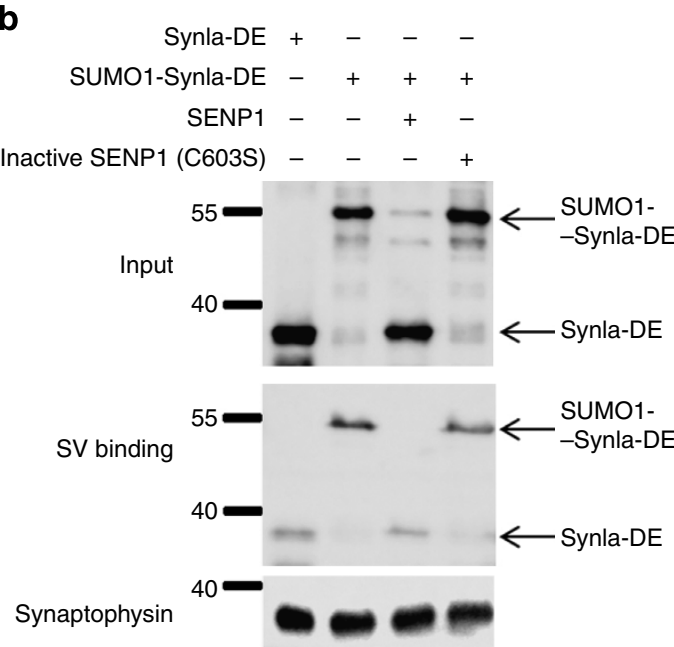

C

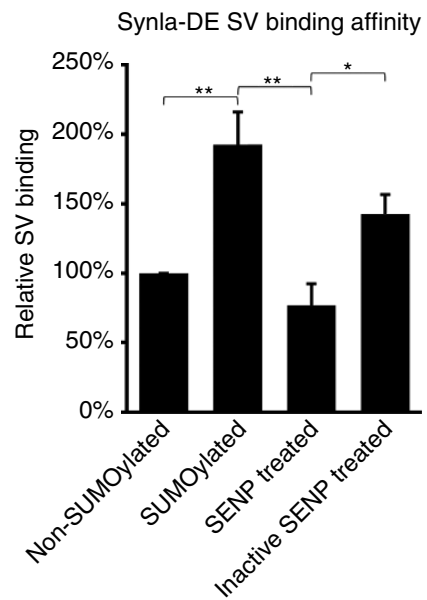

directly bind to the vesicle phospholipid bilayer ${ }^{14,26,27}$. SUMOylation can alter substrate protein conformation ${ }^{28}$ and/or promote protein-protein interactions via SUMO-interacting motifs ${ }^{29}$. Enhanced association of SUMOylated SynIa to SVs may be mediated by (i) SUMOylation-dependent changes in the conformation of SynI, causing an increase in SV affinity and/or by (ii) SUMOylated SynIa binding to a SUMO-interacting motifcontaining SV protein. Moreover, since CamKII phosphorylation promotes the dissociation of SynIa and the release of SVs from the actin cytoskeleton ${ }^{30}$, it is also possible that SUMOylation of SynIa could reduce or prevent CamKII phosphorylation.

SUMOylation is crucial in SynIa targeting and clustering. Because the association of SynIa with SVs is critical for correct localization at the presynaptic terminal ${ }^{31-33}$, we next investigated the effect of SUMOylation on SynIa targeting. We overexpressed the SENP1 catalytic domain fragment in hippocampal neurons to decrease the overall cellular SUMOylation level. Endogenous SynI was then stained together with the presynaptic marker Bassoon. SynI puncta that co-localized with Bassoon were counted and normalized to the total number of SynI puncta ${ }^{34}$. When compared with the overexpression of a catalytically inactive mutant (C603S) of SENP1, there were significantly more SynI puncta that did not co-localize with Bassoon, suggesting that SUMOylation influences the targeting activity of SynI (Fig. 4a,b).

To ensure that the mistargetting effect was specifically due to SynIa SUMOylation, endogenous SynIa was knocked down in rat hippocampal neurons and replaced with either GFP-tagged WT or K687R SynIa, then fixed and the co-localization with bassoon and the SV marker VAMP2 assessed. As shown in Fig. 4c,d, while both WT and K687R SynIa showed punctate distributions, there was significantly lower co-localization of the K687R mutant with Bassoon or VAMP2 compared with WT SynIa. There was no difference in the intensity of VAMP2 staining caused by Syn1a K687R expression (Supplementary Fig. 5a), indicating that SVs themselves are still correctly localized at the active zone when Syn1a SUMOylation is inhibited. These results indicate that preventing SUMOylation causes a defect in presynaptic vesicle targeting of SynIa, resulting in a functional defect in the vesicle pool.

To further explore the role(s) of SUMOylation in SynIa association with SVs, we performed SynIa dispersion and reclustering assays, which measure changes in fluorescence of GFP-tagged SynI at synaptic boutons ${ }^{35}$. Intense synaptic activity causes SynI to diffuse away from synaptic boutons due to dissociation from the SVs. Following stimulation, SynI then reclusters at the synapse. Using this approach, we were able to

Figure 3 | SUMOylation of Synla promotes SV binding. (a) Preparation of SUMOylated SynlaDE fragment. HA-His-SynlaDE was bacterially expressed with or without co-expression of the E1/E2/Flag-SUMO construct ${ }^{44}$. Eluates containing SUMOylated SynlaDE were purified using an anti-Flag antibody resin and eluted with Flag peptide. Representative Coomassiestained SDS-polyacrylamide gel electrophoresis (SDS-PAGE) of the input and eluate from both purification steps are shown. (b) SVs were extracted from rat brain and mixed with purified recombinant HA-His-SynlaDE $(n=6)$ or SUMO-HA-His-SynlaDE $(n=6)$ with active or inactive SENP (both $n=4)$ to de-SUMOylate SynlaDE $(n=4)$. The SVs were isolated by ultracentrifugation, resuspended and analysed by SDS-PAGE and immunoblotting for SynlaDE using anti-HA antibody. Anti-synaptophysin blot (below) shows equal loading of the SV fraction. (c) Quantification of binding of non-modified, SUMOylated, deSUMOylated and inactive SENP-treated SUMOylated SynlaDE to SVs. Data presented as mean \pm s.e.m. ${ }^{\star \star} P<0.01,{ }^{\star} P<0.05$ one-way ANOVA, post hoc test with Bonferroni correction. $n$ numbers as above 
a

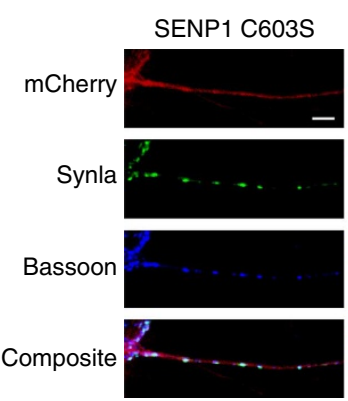

c

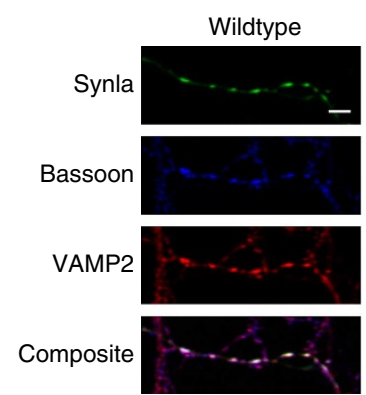

e

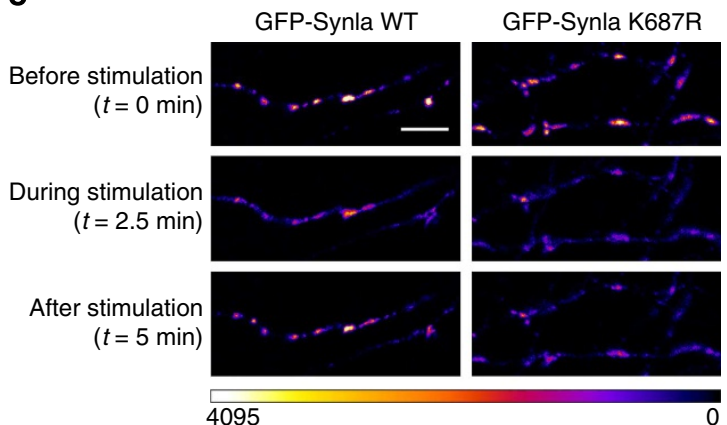

$\mathbf{f}$

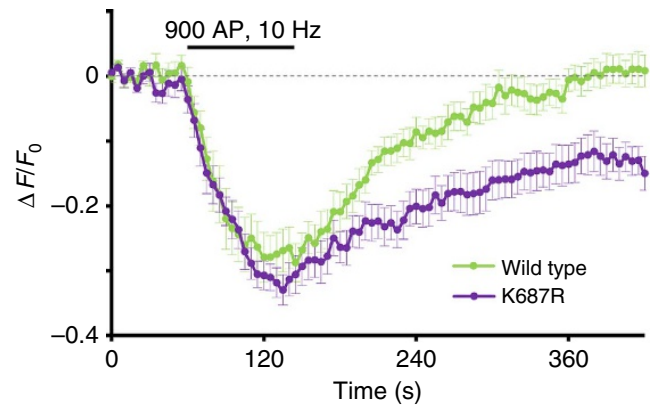

b

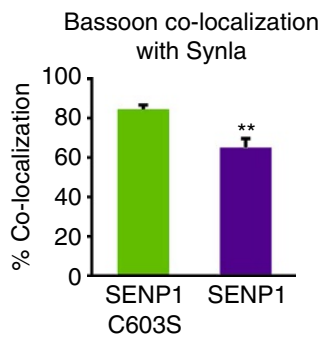

d
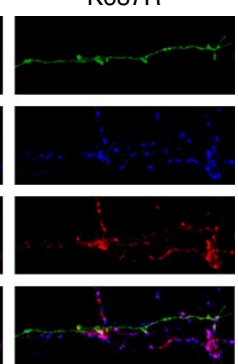

g

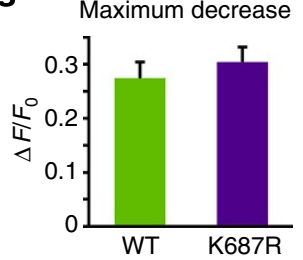

h

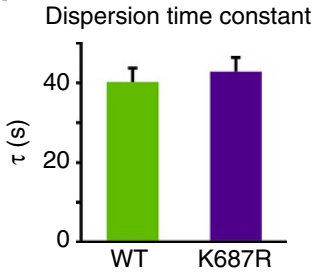

i Reclustering time constant

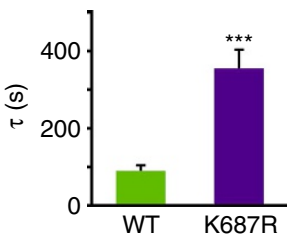

Figure 4 | SUMOylation of Synla modulates its presynaptic targeting and reclustering at the presynapse following stimulation. (a) SUMOylation is required for presynaptic targeting. WT $(n=12)$ or C603S (dead mutant, $n=12)$ SENP1-mCherry was overexpressed in DIV 9-10 hippocampal neurons. Cells were fixed 7 days later and immunostained with anti-Synl and anti-bassoon (presynaptic marker) antibodies. Scale bar, $5 \mu \mathrm{m}$. (b) Quantification of a. Co-localizing Synla and bassoon-positive puncta were counted and divided by the total number of puncta. Data presented as mean \pm s.e.m. ${ }^{\star \star} P<0.01$ against C603S mutant, unpaired Student's t-test. (c) SUMOylation of Syn1a is required for correct presynaptic targeting. Molecular replacement of endogenous Synla in hippocampal neurons with GFP-Synla WT $(n=13)$ or GFP-Synla K687R $(n=13)$. Neurons were transfected at DIV 9-10 and fixed and imaged 7 days later using anti-bassoon as an active zone marker, and anti-VAMP2 as SV marker. Scale bar, $5 \mu$ m. (d) Quantification of c. Synaptic co-localization of Synla with bassoon or VAMP2 positive puncta were counted and divided by the total number of puncta. Data presented as mean \pm s.e.m. ${ }^{\star \star \star} P<0.001$ against wild type with respect to the same marker, unpaired Student's $t$-test. (e) DIV 9-10 hippocampal neurons transfected with wild type $(n=9)$ or K687R $(n=12)$ GFP-tagged Synla and imaged 7 days later. Electric field stimulation $(900 \mathrm{AP}$ at $10 \mathrm{~Hz})$ was applied after $60 \mathrm{~s}$ of baseline recording and the recovery followed for $300 \mathrm{~s}$ afterwards. Representative images of clusters of GFP-Synla WT and GFP-Synla K687R before, during and after stimulation. Colour scale in arbitrary fluorescent units. Scale bar, $5 \mu \mathrm{m}$. (f) Dispersion and reclustering profiles GFP-Synla WT and GFP-Synla K6887R fluorescence. The data are presented as mean \pm s.e.m. of the changes in punctate fluorescence $\left(\Delta F / F_{0}\right)$. $n$ numbers as in e. (g) Histogram showing the mean \pm s.e.m. of maximum dispersion at $t=180 \mathrm{~s}$. $n$ numbers as in $\mathbf{e}$. $(\mathbf{h}, \mathbf{i})$ The dispersion $(t=60-150 \mathrm{~s})$ and reclustering $(t=150-420 \mathrm{~s})$ profiles were fitted with exponential curves and time constants presented as mean \pm s.e.m. ${ }^{\star \star \star} P<0.001$ against wild type, Student's $t$-test. $n$ numbers as in e. 
a

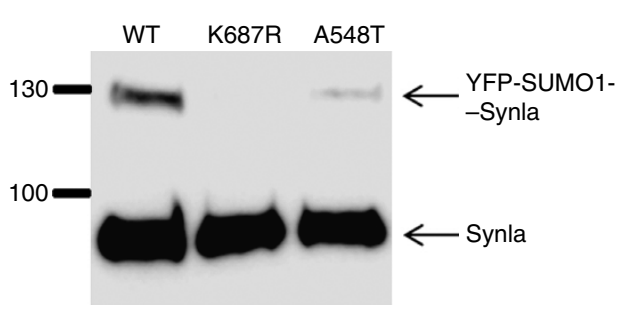

C

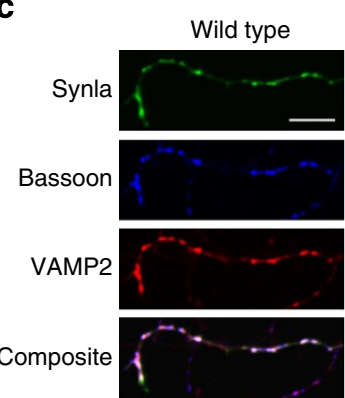

e

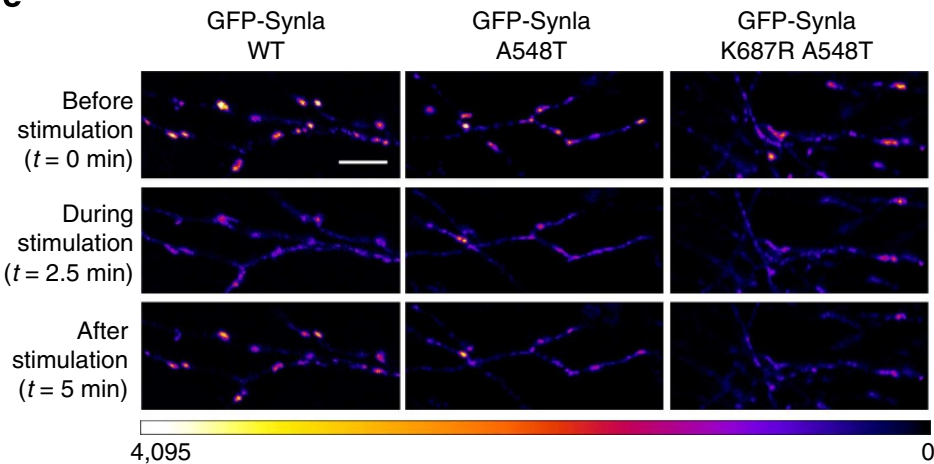

f

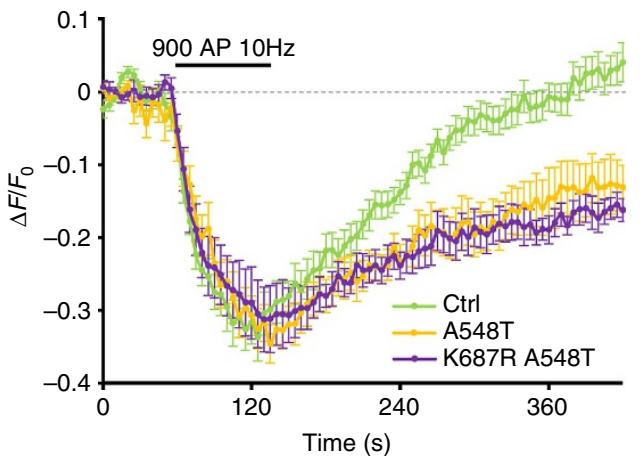

b

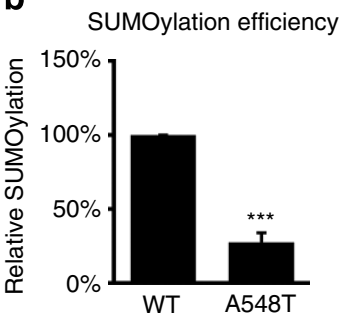

d
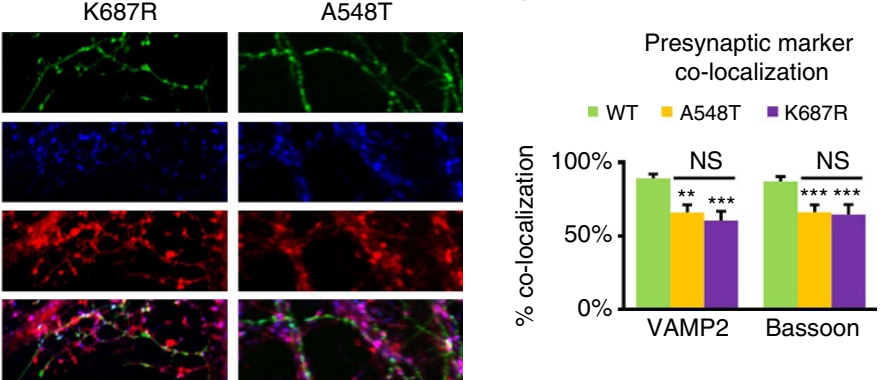

g

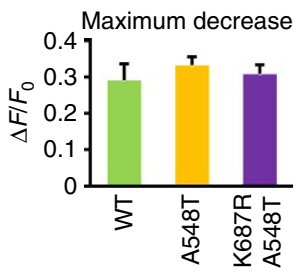

h Dispersion time constant

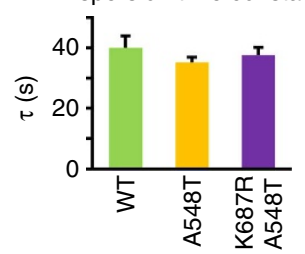

i

Reclustering time constant

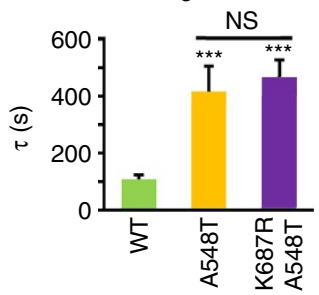

Figure 5 | The ASD-associated A548T mutation impairs Synla SUMOylation. (a) Immunoblot showing reduced SUMOylation of HA-Synla A548T in N2A cells. (b) Quantitative analysis of the extent of HA-Synla A548T SUMOylation. The blots were quantified by taking the ratio of SUMOylated band to the unmodified band normalized to HA-Synla WT, data presented as mean \pm s.e.m. ${ }^{\star \star} P<0.01$ unpaired Student's $t$-test, $n=6$. (c) Representative images showing effects of molecular replacement of endogenous Synla with GFP-Synla WT $(n=13)$, GFP-Synla A548T $(n=13)$ and GFP-Synla K687R $(n=13)$ in hippocampal neurons. Neurons were stained with anti-bassoon as an active zone marker or anti-VAMP2 as SV marker. Scale bar, $5 \mu \mathrm{m}$ (d) Quantitative analysis of synaptic co-localization as described in Fig. 4c. Data presented as mean \pm s.e.m. ${ }^{\star \star \star} P<0.001$ against wild type; NS, not significant, One-way ANOVA, Bonferroni correction and post hoc test (e) Representative images of the dispersion and reclustering assay for GFP-Synla WT, GFP-Synla A548T and GFP-Synla K687R-A548T. Scale bar, $5 \mu \mathrm{m}$. Colour scale in arbitrary fluorescence units. (f) Image analysis of e using $\Delta F / F_{0}$ plotted against time to give the dispersion and reclustering profile. Mean \pm s.e.m. of GFP-Synla WT $(n=10)$, GFP-Synla A548T $(n=14)$ and GFP-Synla K687R-A548T ( $n=13)$.

(g) Histogram showing the mean \pm s.e.m. of maximum dispersion at $t=150 \mathrm{~s}$. $n$ numbers as in $\mathbf{f}$. (h,i) The dispersion ( $t=60-150 \mathrm{~s})$ and reclustering $(t=150-420 \mathrm{~s})$ profiles were fitted with exponential curves and time constants presented as mean \pm s.e.m. ${ }^{\star \star \star} P<0.001$ against $W T ; N S$, not significant, one-way ANOVA, post hoc test with Bonferroni correction. $n$ numbers as in $\mathbf{f}$. 
determine the contribution of SUMOylation to both association and disassociation of SynIa with SVs.

We expressed GFP-tagged SynIa WT and K687R mutant in hippocampal neurons. At rest, both proteins displayed a clear punctate distribution. Electrical field stimulation of $900 \mathrm{AP}$ at $10 \mathrm{~Hz}$ produced a significant decrease in punctate fluorescence as GFP-SynIa diffused away from synaptic boutons (Fig. 4e,f). Furthermore, WT and K687R SynIa showed a similar magnitude and time constant of dispersion (Fig. 4g,h), as well as the number of responsive puncta (Supplementary Fig. 5b). Importantly, however, the K687R mutant exhibited markedly different reclustering dynamics. Punctate fluorescence for WT SynIa completely recovered to the baseline levels $3 \mathrm{~min}$ after stimulation, whereas the non-SUMOylatable K687R mutant only recovered $\sim 50 \%$ at $3 \mathrm{~min}$ and had a significantly higher time constant for reclustering (Fig. 4i). These results indicate that SUMOylation does not participate in SynIa dissociation from SVs but that it is required for reclustering after synaptic activation. Furthermore, because CamKII phosphorylation mediates SynIa dissociation from SVs, these data argue against a direct interplay between phosphorylation and SUMOylation of SynIa in regulating SynIa-SV interactions. Rather, our results suggest that phosphorylation and SUMOylation control SynIa function via separately coordinated pathways.

Taken together, our data indicate that SUMOylation of SynIa promotes binding to SVs and that this is required for correct presynaptic targeting of SynIa and reclustering of SVs following stimulation. This, in turn, replenishes and maintains the reserve vesicle pool to ensure presynaptic integrity and correct dynamics of neurotransmitter release. We attribute the decrease in SV exocytosis when SynIa SUMOylation is inhibited (Fig. 2) to the defects in the establishment and maintenance of the vesicle pools, resulting in fewer vesicles being available for release during prolonged stimulation. In particular, this phenotype bears striking resemblance to that observed in sypHy exocytosis assay under cdk 5 inhibition ${ }^{5}$, in which a shift in SV ratio between releasable and non-releasable pool was observed. One attractive possibility is that SUMOylation and cdk5 are part of a pathway that fine-tunes the ratio of releasable and non-releasable SVs.

As reported for most SUMO substrates ${ }^{36}$, only a small proportion of SynIa is SUMOylated at any given time. Consistent with this, our proposed mechanism of action only requires SynIa to be SUMOylated for the initiation of SV association. Once the binding is stabilized, SUMO can be removed as SynIa itself has a high affinity for SV. (Graphical representation in Supplementary Fig. 6).

SynIa SUMOylation and disease. Mutations in SynIa have been associated with epilepsy and $\mathrm{ASD}^{37}$. These include two nonsense mutations that abolish the $\mathrm{E}$ domain and other less severe mutations that do not significantly affect SV binding or phosphorylation by CamKII, but do result in defective SynIa targeting and SV exocytosis ${ }^{34}$. We therefore investigated the possible role of SUMOylation in the pathogenesis of these mutations. Of the mutants we investigated, the A548T mutation (analogous to A550T in human SynIa ${ }^{34}$ ) significantly decreased the levels of SynIa SUMOylation in N2A cells (Fig. 5a,b), and the defect in SV exocytosis caused by the A548T mutation is strikingly similar to that observed with K687R mutation ${ }^{34}$, suggesting a link between SUMOylation and ASD/epilepsy.

We next compared the presynaptic localization of GFP-SynIa WT, K687R and A548T in neurons in which endogenous SynIa was knocked down (Fig. 5c). Both non-SUMOylatable K687R and the autism mutant A548T exhibited similar SynIa targeting defects compared with WT SynIa (Fig. 5d). While we cannot formally exclude the possibility that targeting defects result from disruption of distinct pathways, these results are consistent with a common mechanism of action. To explore this in more detail, we used the SynIa dispersion assay to compare the effects of the A548T with a double A548T/K687R mutant. As expected, there were no differences in the dynamics of dispersion between the WT and either mutant, but both mutants were significantly slower in reclustering compared with WT. Moreover, the time constants for reclustering were identical for the A548T and the A548T/K687R double mutant (Fig. 5e-i) indicating that there is no additive effect of the A548T mutation in non-SUMOylatable SynIa. Again, these data are consistent with both A548T and K687R disrupting SynIa reclustering via the same pathway, and strongly suggest that the defects in SV exocytosis and SynIa targeting/clustering in this ASD mutation can be attributed to defective SUMOylation.

How the A548T mutation affects SUMOylation at the molecular level remains to be determined. Residues A548 and K687 are relatively distant from each other and in silico analysis of the A548T mutation does not predict any marked alteration in SynIa structure ${ }^{34}$. Interestingly, an in vitro SUMOylation assay showed no decrease in SUMOylation efficiency for A548T mutant (Supplementary Fig. 7). Since SUMOylation in vitro requires only Ubc9 interaction, it is unlikely that A548T mutation directly influences Ubc9 binding. Nonetheless, one possible explanation is that A548T changes SynIa conformation in such a way to occlude the SUMO consensus sequence. Alternatively, A548 may be required for SynIa interactions with a SUMO E3 ligase. Overall, our data clearly support the hypothesis that the A548T mutation-mediated defects in SynIa SUMOylation result in the dysfunction of SV dynamics. These changes, in turn, likely cause imbalances between excitatory and inhibitory stimulation that can underlie ASD and epilepsy ${ }^{38-40}$. Thus, we propose that SynIa SUMOylation plays a critical role in presynaptic function and dysfunction and that modulation of this pathway represents a potentially powerful strategy for the development of new approaches to treating synaptopathies including ASD and epilepsy.

\section{Conclusion}

Here we show that SUMOylation of SynIa enhances its SV reclustering at the $\mathrm{RP}$, which is required to maintain presynaptic responsiveness and normal synaptic transmission. We further demonstrate that the A548T mutation in SynIa reduces SUMOylation and that this may account for the epilepsy and ASD phenotype associated with this mutation. These results expand the repertoire of synaptic proteins that are regulated by SUMOylation and provide further evidence for the fundamentally important roles of SUMO modification of synaptic proteins in health and disease.

\section{Methods}

Molecular biology. For knockdown-rescue experiments, shRNA-resistant SynIa constructs were expressed as mCherry/GFP-IRES-SynIa cassette on a pFIV plasmid, which also expressed SynI shRNA (vector made in house, shRNA target sequence $5^{\prime}$-CCATGGAGAAATTGACATTAATA- $3^{\prime}$ against SynI). For all knockdown studies, 'Control' cells were transfected with pFIV (expressing mCherry or GFP) without shRNA. The Flag-tagged SUMOylation vector used in bacterial SUMOylation of SynIaDE are engineered by inserting Flag tag at the $\mathrm{N}$ terminus of SUMO in pE1E2S1 vector. All subcloning and mutagenesis reactions were performed according to standard protocols.

Cell culture. Embryonic cortical and hippocampal neurons were isolated from E18 Wistar Rattus Norvergicus embryos. Brain areas were dissected and trypsinized, before being plated on either PLL-coated 25-mm glass coverslips (for hippocampal cells) or PLL-coated six-well plates (for cortical cells). Cells were initially plated in plating media (Neurobasal media $+10 \%$ horse serum, B27 supplement, $2 \mathrm{mM}$

Glutamax, $1 \times$ penicillin/streptomycin), which after $24 \mathrm{~h}$ was replaced with feeding 
media (Neurobasal media, B27 supplement, $1.2 \mathrm{mM}$ Glutamax, $1 \times$ penicillin/ streptomycin). For more details, see ref. 41. For biochemistry, cortical neurons were used at DIV18. For SypHy experiments, hippocampal neurons are transfected at DIV9-10 and imaged 5 days later. For dispersion/reclustering assay and localization experiments, hippocampal neurons are transfected at DIV9-10 and imaged 7 days later. All neuronal transfections were performed using Lipofectamine 2000 (Invitrogen) according to manufacturer's instructions.

Biochemistry. SUMO-1 co-immunoprecipitation was performed from whole rat brain lysate. Brain tissue $(250 \mathrm{mg})$ was flash frozen and pulverized under liquid nitrogen. The resulting powder was dissolved in high SDS lysis buffer $(25 \mathrm{mM}$ HEPES, $500 \mathrm{mM} \mathrm{NaCl}$, $1 \%$ Triton X-100, $2 \%$ SDS, $0.5 \%$ Sodium deoxycholate, $\mathrm{pH}$ 7.4) and sonicated. The lysate was subsequently diluted 10 -fold with RIPA dilution buffer (25 mM HEPES, $500 \mathrm{mM} \mathrm{NaCl}, 1 \%$ Triton X-100, 0.5\% Sodium deoxycholate, $\mathrm{pH}$ 7.4), which was used for pull down with $4 \mu \mathrm{g}$ of anti-SUMO-1 D11 antibody (Santa Cruz, \# sc-5308) bound to Protein A/G magnetic beads (Pierce). Western blotting was performed using anti-SynI antibody at 1:5,000 (BD biosciences, \#611392).

For the N2A SUMOylation assay ${ }^{42}$, cells were transfected with HA-SynIa, Flag-Ubc9 and either active YFP-SUMO-1-GG or non-conjugatable YFP-SUMO$\Delta \mathrm{GG}$ for $48 \mathrm{~h}$ before lysis on ice in buffer containing $20 \mathrm{nM}$ NEM. GFP-Trap (Chromotek) was used for GFP-tag pull down according to the manufacturer's instructions. Western blotting was performed using rat anti-HA antibody at 1:1,000 (Roche, \# 11583816001)

For GST-Ubc9 pull-down assay, GST-Ubc9 was expressed in BL21(DE3) E. coli under standard isopropylthiogalactoside induction. Harvested bacteria were lysed (lysis buffer: $25 \mathrm{mM}$ HEPES, $500 \mathrm{mM} \mathrm{NaCl}, 2 \mathrm{mM}$ DTT, $1 \%$ Triton X-100) and incubated with glutathione resin (GE Healthcare). After washing extensively, the resin was then incubated with cell lysate from cortical neuronal culture or HEK293T in lysis buffer, obtained by resuspending and sonicating cells in lysis buffer (25 mM HEPES, $150 \mathrm{mM} \mathrm{NaCl}, 1 \%$ Triton X-100, pH 7.4). Western blotting was performed using anti-SynI (for neuronal lysate) or anti-HA antibody (for HEK293T lysate).

Where cropping of western blots was performed, the full blots are supplied (Supplementary Fig. 8), with boxed areas indicating the cropped areas used.

Bacterial expression and purification of Synla constructs. Bacterial expression of His-HA-SynIaDE was carried out in BL21(DE3) E. coli using a standard isopropylthiogalactoside overnight induction protocol. The harvested bacteria were lysed by sonication in Nickel-Binding Buffer $(25 \mathrm{mM}$ HEPES, $500 \mathrm{mM} \mathrm{NaCl}$, $20 \mathrm{mM}$ Imidazole, protease inhibitors, $\mathrm{pH}$ 7.4). Precleared bacterial lysate was then load onto Ni-NTA resin. After extensive washes, the protein was eluted with elution buffer ( $25 \mathrm{mM}$ HEPES, $200 \mathrm{mM}$ imidazole, $\mathrm{pH}$ 7.4), then concentrated to $2 \mathrm{mg} \mathrm{ml}^{-1}$ before snap freezing for storage at $-80^{\circ} \mathrm{C}$. SUMOylated SynIaDE was produced by co-expression of His-HA-SynIaDE and Flag-SUMOylation plasmid in BL21(DE3) E. coli and metal-affinity purification described above and the eluate loaded onto a Flag-affinity gel (SIGMA). Retained proteins were eluted using $50 \mathrm{\mu g} \mathrm{ml}^{-1}$ Flag peptide (SIGMA) in $25 \mathrm{mM}$ HEPES, pH 7.4. Eluates were then snap-frozen for storage at $-80^{\circ} \mathrm{C}$.

SV-binding assay. Whole rat brain was homogenized in $10 \mathrm{ml}$ ice-cold buffered sucrose (4 mM HEPES, $320 \mathrm{mM}$ Sucrose, pH 7.3) using a glass-Teflon homogenizer. The homogenate was then centrifuged in a fixed angle rotor at $800 \mathrm{~g}$ for $10 \mathrm{~min}$. The supernatant was centrifuged at $9,200 \mathrm{~g}$ for $15 \mathrm{~min}$ and then the subsequent supernatant was discarded. The pellet was resuspended with $10 \mathrm{ml}$ of buffered sucrose and then centrifuged at $10,200 \mathrm{~g}$ for $15 \mathrm{~min}$. The supernatant was discarded and the pellet was resuspended in $1 \mathrm{ml}$ of buffered sucrose. Ice-cold water $(9 \mathrm{ml})$ was then added and the suspension was homogenized with a glass-Teflon homogenizer briefly at maximum speed. HEPES-NaOH (1 M, pH 7.4) was added to the suspension to a final concentration of $3 \mathrm{mM}$, and was incubated on ice for $30 \mathrm{~min}$, then centrifuged at $25,000 \mathrm{~g}$ for $20 \mathrm{~min}$. The pellet, containing crude SVs, was retained. For more details, see ref. 43. SVs were resuspended in $10 \mathrm{ml}$ buffered sucrose ( $5 \mathrm{mM}$ HEPES, pH 7.4, $300 \mathrm{mM}$ Sucrose) and $2.5 \mathrm{ml}$ of SV suspension was mixed with either $3 \mu \mathrm{g}$ of SUMOylated or non-SUMOylated/ SENP-deSUMOylated His-HA-SynIa-DE and incubated at $4{ }^{\circ} \mathrm{C}$ with gentle agitation for $1 \mathrm{~h}$. The SVs were then collected by ultracentrifugation at $55,000 \mathrm{~g}$ for $2 \mathrm{~h}$, resuspended in $200 \mu \mathrm{l}$ buffered sucrose and the extent of His-HA-SynIa-DE binding was assessed by western blot analysis using an anti-HA antibody. SENP treatment of SUMO-SynIaDE was performed by adding purified recombinant SENP (catalytic domain only) at 1:100 mass ratio to SUMOylated His-HASynIaDE at incubate at room temperature for $30 \mathrm{~min}$.

Immunocytochemistry. Immunocytochemistry was performed following paraformaldehyde fixation of cells and permeabilization with $0.1 \%$ Triton X-100. For presynaptic co-localization assays, anti-bassoon at 1:500 (Abcam, \#ab82958) and anti-VAMP2 at 1:1,000 (Synaptic Systems, \#104202) was used to stain the presynaptic terminal. The number of GFP puncta positive for bassoon or VAMP2 were counted and reported as a percentage of total GFP puncta. Each field is counted as one $n$ and consists of at least 20 puncta and at least 3 independent neuronal culture preparations were assessed. For quantification in the knockdownrescue experiments, anti-SynI at 1:500 (Novua \# NB300-104) was used to assess SynI levels in mCherry-expressing neurons. All quantification was performed using ImageJ software.

SypHy experiments. Neurons co-transfected with SynIa knockdown, or knockdown-rescue constructs (see Molecular Biology section for details) were mounted in a Warner stimulation chamber (Harvard Apparatus), connected to a Digitimer Constant Voltage stimulator and a Master-8 pulse train generator. Exocytosis was triggered using electrical field stimulation, using $1 \mathrm{~ms}$ pulses (APs) of $50 \mathrm{~V}$ with a paradigm of $600 \mathrm{APs}$ at $20 \mathrm{~Hz}$. All experiments were performed in HBS with $50 \mu \mathrm{M}$ D-AP5, $25 \mu \mathrm{M}$ CNQX and $1 \mu \mathrm{M}$ bafilomycin A. Perfusion with $\mathrm{NH}_{4} \mathrm{Cl}$ (pH 7.4), was used to reveal total levels of SypHy loading at the end of each experiment. Images were taken at $0.5 \mathrm{~Hz}$ using a CCD camera with a GFP filter cube. For each cell, 10 regions of interest (ROIs) with area of $2.13 \times 2.13 \mu \mathrm{m}$ were manually placed covering single SypHy puncta chosen blindly. Average fluorescence signal were measured with non-responsive ROIs discounted. Data were first normalized to background levels (that is, $\Delta F / F_{0}$ ), and then expressed as a percentage of fluorescence after $\mathrm{NH}_{4} \mathrm{Cl}$ perfusion $\left(F_{\max }\right)$ or after stimulation $\left(F_{\text {post-stim }}\right)$. Extrasynaptic ROIs were used to correct for background fluorescence. For more details, please see ref. 17

Synla dispersion and reclustering assay. GFP-SynIa-transfected neurons were mounted in the electrical field stimulation chamber, filled with HBS supplemented with $25 \mu \mathrm{M} \mathrm{APV}$ and $50 \mu \mathrm{M}$ CNQX. Confocal images were recorded at $0.2 \mathrm{~Hz}$ for $10 \mathrm{~min}$ using $488 \mathrm{~nm}$ argon laser excitation, with a 500-530-nm band-pass filter. Images were scanned at $512 \times 512$ resolution with suitable gain and offset for the best contrast and avoiding saturation of signals. This recording was to establish the effect of photobleaching, and therefore no stimulation was performed. After the recording was finished, a nearby region of the same neuron was imaged with identical settings. For this recording, $900 \mathrm{AP}$ at $10 \mathrm{~Hz}(90 \mathrm{~s})$ electrical stimulation $(50 \mathrm{~V}, 1 \mathrm{~ms}$ pulses) were delivered $1 \mathrm{~min}$ after the start of image recording. For each cell, seven ROIs with area of $0.83 \times 0.83$ were manually placed covering single GFP puncta, chosen blindly at $t=0$. Average fluorescence signals were measured with non-responsive ROIs discounted. Changes in fluorescence from initial values were normalized to initial levels (that is, $\Delta F / F_{0}$ ). The same analysis was performed on the unstimulated recording to correct for photobleaching. To calculate the dispersion rate, the $\Delta F / F_{0}$ at $t=60-150 \mathrm{~s}$ of each neuron were fitted with a single-fall exponential decay as follows

$$
y=-A\left(1-e^{-t / \tau}\right)
$$

where $A$ is the plateau of the curve and $\tau$ is the time constant that reflects the rate of increase. The $\tau$ from neurons transfected with the same construct were averaged for comparison between constructs with statistical testing.

To calculate the reclustering rate, the $\Delta F / F_{0}$ at $t=150-600 \mathrm{~s}$ of each neuron were fitted with a single-fall exponential decay as follows

$$
y=A\left(1-e^{t / \tau}\right)
$$

where $A$ is the plateau of the curve and $\tau$ is the time constant that reflect the rate of increase. The $\tau$ from neurons transfected with the same construct were averaged and then compared between different constructs with statistical testing.

For further details, see ref. 35 .

Statistical analysis. Graphpad Prism software (Graphpad Inc.) was used for statistical analysis. Data were analysed using one-way analysis of variance (ANOVA) with Bonferroni's post hoc test (for comparisons between multiple data sets) or two-tailed Student's $t$-test (comparison between two data sets). Curve fitting in Figs 2, 4 and 5 was performed using Graphpad Prism, fitting to a single exponential rise function $y=a\left(1-e^{-t / \tau}\right)$ where $\tau$ is the time constant.

Sequence alignment. Sequence alignment is done in UGENE using ClustalX algorithm. Synapsins sequences were obtained from NCBI.

\section{References}

1. Rizzoli, S. O. \& Betz, W. J. Synaptic vesicle pools. Nat. Rev. Neurosci. 6, 57-69 (2005).

2. Ceccaldi, P. E. et al. Dephosphorylated synapsin I anchors synaptic vesicles to actin cytoskeleton: an analysis by videomicroscopy. J. Cell Biol. 128, 905-912 (1995).

3. Cesca, F., Baldelli, P., Valtorta, F. \& Benfenati, F. The synapsins: key actors of synapse function and plasticity. Prog. Neurobiol. 91, 313-348 (2010).

4. Bykhovskaia, M. Synapsin regulation of vesicle organization and functional pools. Semin. Cell Dev. Biol. 22, 387-392 (2011).

5. Verstegen, A. M. et al. Phosphorylation of synapsin I by cyclin-dependent kinase- 5 sets the ratio between the resting and recycling pools of synaptic vesicles at hippocampal synapses. J. Neurosci. 34, 7266-7280 (2014). 
6. Jovanovic, J. N. et al. Opposing changes in phosphorylation of specific sites in synapsin I during $\mathrm{Ca} 2+$-dependent glutamate release in isolated nerve terminals. J. Neurosci. 21, 7944-7953 (2001).

7. Wilkinson, K. A., Nakamura, Y. \& Henley, J. M. Targets and consequences of protein SUMOylation in neurons. Brain Res. Rev. 64, 195-212 (2010).

8. Craig, T. J. \& Henley, J. M. Protein SUMOylation in spine structure and function. Curr. Opin. Neurobiol. 22, 480-487 (2012).

9. Luo, J. et al. Receptor trafficking and the regulation of synaptic plasticity by SUMO. Neuromol. Med. 15, 692-706 (2013).

10. Feligioni, M., Nishimune, A. \& Henley, J. M. Protein SUMOylation modulates calcium influx and glutamate release from presynaptic terminals. Eur. J. Neurosci. 29, 1348-1356 (2009).

11. Guo, C. \& Henley, J. Wrestling with stress: roles of protein SUMOylation and deSUMOylation in cell stress response. IUBMB Life 66, 71-77 (2014).

12. Girach, F., Craig, T. J., Rocca, D. L. \& Henley, J. M. RIM1alpha SUMOylation is required for fast synaptic vesicle exocytosis. Cell Rep. 5, 1294-1301 (2013).

13. Hilfiker, S. et al. Two sites of action for synapsin domain $\mathrm{E}$ in regulating neurotransmitter release. Nat. Neurosci. 1, 29-35 (1998).

14. Monaldi, I. et al. The highly conserved synapsin domain E mediates synapsin dimerization and phospholipid vesicle clustering. Biochem. J. 426, 55-64 (2010).

15. Sudhof, T. C. et al. Synapsins: mosaics of shared and individual domains in a family of synaptic vesicle phosphoproteins. Science 245, 1474-1480 (1989).

16. Candiani, S. et al. The synapsin gene family in basal chordates: evolutionary perspectives in metazoans. BMC Evol. Biol. 10, 32 (2010).

17. Burrone, J., Li, Z. \& Murthy, V. N. Studying vesicle cycling in presynaptic terminals using the genetically encoded probe synaptopHluorin. Nat. Protoc. 1, 2970-2978 (2006)

18. Ikeda, K. \& Bekkers, J. M. Counting the number of releasable synaptic vesicles in a presynaptic terminal. Proc. Natl Acad. Sci USA 106, 2945-2950 (2009).

19. Li, L. et al. Impairment of synaptic vesicle clustering and of synaptic transmission, and increased seizure propensity, in synapsin I-deficient mice. Proc. Natl Acad. Sci. USA 92, 9235-9239 (1995).

20. Rosahl, T. W. et al. Essential functions of synapsins I and II in synaptic vesicle regulation [see comments]. Nature 375, 488-493 (1995).

21. Ryan, T. A., Li, L., Chin, L. S., Greengard, P. \& Smith, S. J. Synaptic vesicle recycling in synapsin I knock-out mice. J. Cell Biol. 134, 1219-1227 (1996).

22. Buchner, A. et al. Sumoylation of p35 modulates p35/cyclin-dependent kinase (Cdk) 5 complex activity. Neuromol. Med. 17, 12-23 (2015).

23. Geerts, C. J., Jacobsen, L., van de Bospoort, R., Verhage, M. \& Groffen, A. J. Tomosyn interacts with the SUMO E3 ligase PIASgamma. PLoS ONE 9, e91697 (2014).

24. Uchimura, Y., Nakamura, M., Sugasawa, K., Nakao, M. \& Saitoh, H. Overproduction of eukaryotic SUMO-1- and SUMO-2-conjugated proteins in Escherichia coli. Anal. Biochem. 331, 204-206 (2004).

25. Wilkinson, K. A., Nishimune, A. \& Henley, J. M. Analysis of SUMO-1 modification of neuronal proteins containing consensus SUMOylation motifs. Neurosci. Lett. 436, 239-244 (2008).

26. Benfenati, F., Bahler, M., Jahn, R. \& Greengard, P. Interactions of synapsin I with small synaptic vesicles: distinct sites in synapsin I bind to vesicle phospholipids and vesicle proteins. J. Cell Biol. 108, 1863-1872 (1989).

27. Benfenati, F., Valtorta, F., Bahler, M. \& Greengard, P. Synapsin I, a neuronspecific phosphoprotein interacting with small synaptic vesicles and F-actin. Cell Biol. Int. Rep. 13, 1007-1021 (1989).

28. Gareau, J. R. \& Lima, C. D. The SUMO pathway: emerging mechanisms that shape specificity, conjugation and recognition. Nat. Rev. Mol. Cell Biol. 11, 861-871 (2011).

29. Kerscher, O. SUMO junction-what's your function? New insights through SUMO-interacting motifs. EMBO Rep. 8, 550-555 (2007).

30. Benfenati, F., Neyroz, P., Bahler, M., Masotti, L. \& Greengard, P. Time-resolved fluorescence study of the neuron-specific phosphoprotein synapsin I. Evidence for phosphorylation-dependent conformational changes. J. Biol. Chem. 265, 12584-12595 (1990).

31. Gitler, D. et al. Different presynaptic roles of synapsins at excitatory and inhibitory synapses. J. Neurosci. 24, 11368-11380 (2004).
32. Tang, Y. et al. Fast vesicle transport is required for the slow axonal transport of synapsin. J. Neurosci. 33, 15362-15375 (2013).

33. Easley-Neal, C., Fierro, J. Jr., Buchanan, J. \& Washbourne, P. Late recruitment of synapsin to nascent synapses is regulated by Cdk5. Cell Rep. 3, 1199-1212 (2013).

34. Fassio, A. et al. SYN1 loss-of-function mutations in autism and partial epilepsy cause impaired synaptic function. Hum. Mol. Genet. 20, 2297-2307 (2011).

35. Chi, P., Greengard, P. \& Ryan, T. A. Synapsin dispersion and reclustering during synaptic activity. Nat. Neurosci. 4, 1187-1193 (2001).

36. Wilkinson, K. A. \& Henley, J. M. Mechanisms, regulation and consequences of protein SUMOylation. Biochem. J. 428, 133-145 (2010).

37. Garcia, C. C. et al. Identification of a mutation in synapsin I, a synaptic vesicle protein, in a family with epilepsy. J. Med. Genet. 41, 183-186 (2004).

38. Etholm, L. \& Heggelund, P. Seizure elements and seizure element transitions during tonic-clonic seizure activity in the synapsin I/II double knockout mouse: a neuroethological description. Epilepsy Behav. 14, 582-590 (2009).

39. Greco, B. et al. Autism-related behavioral abnormalities in synapsin knockout mice. Behav. Brain Res. 251, 65-74 (2013).

40. Lignani, G. et al. Epileptogenic Q555X SYN1 mutant triggers imbalances in release dynamics and short-term plasticity. Hum. Mol. Genet. 22, 2186-2199 (2013).

41. Martin, S. \& Henley, J. M. Activity-dependent endocytic sorting of kainate receptors to recycling or degradation pathways. EMBO J. 23, 4749-4759 (2004)

42. Craig, T. J. \& Henley, J. M. SUMOylation, Arc and the regulation homeostatic synaptic scaling: implications in health and disease. Commun. Integr. Biol. 5, 634-636 (2012).

43. Huttner, W. B., Schiebler, W., Greengard, P. \& De Camilli, P. Synapsin I (protein I), a nerve terminal-specific phosphoprotein. III. Its association with synaptic vesicles studied in a highly purified synaptic vesicle preparation. J. Cell Biol. 96, 1374-1388 (1983).

44. Uchimura, Y., Nakao, M. \& Saitoh, H. Generation of SUMO-1 modified proteins in E. coli: towards understanding the biochemistry/structural biology of the SUMO-1 pathway. FEBS Lett. 564, 85-90 (2004).

\section{Acknowledgements}

We are grateful to the MRC, BBSRC and ERC for financial support, to Dr Kevin Wilkinson for advice and critical reading of the manuscript and to Philip Rubin for expert technical support and assistance.

\section{Author contributions}

L.T.-H.T. performed virtually all of the experimental work and analysis, and also assisted with experimental design and writing the manuscript. T.J.C. directed the project, developed protocols, performed some of the SypHy imaging and assisted writing of the manuscript. J.M.H. oversaw the project and writing of the manuscript and obtained funding.

\section{Additional information}

Supplementary Information accompanies this paper at http://www.nature.com/ naturecommunications

Competing financial interests: The authors declare no competing financial interests.

Reprints and permission information is available online at http://npg.nature.com/ reprintsandpermissions/

How to cite this article: Tang, L. T.-H. et al. SUMOylation of Synapsin Ia maintains synaptic vesicle availability and is reduced in an autism mutation. Nat. Commun. 6:7728 doi: $10.1038 /$ ncomms8728 (2015).

This work is licensed under a Creative Commons Attribution 4.0 International License. The images or other third party material in this article are included in the article's Creative Commons license, unless indicated otherwise in the credit line; if the material is not included under the Creative Commons license, users will need to obtain permission from the license holder to reproduce the material. To view a copy of this license, visit http://creativecommons.org/licenses/by/4.0/ 